\title{
WRAPPING OF THE MODERATELY DILATED ASCENDING AORTA BY FRESH AUTOLOGOUS PERICARDIUM
}

\author{
Antonio Calafiore ${ }^{1}$, Sotirios Prapas $^{1}$, Kostas katsavrias ${ }^{1}$, Michele Di Mauro ${ }^{2}$, Panayiotis \\ Zografos $^{1}$, Stefano Guarracini ${ }^{3}$, and Stella Papandreopoulou ${ }^{1}$ \\ ${ }^{1}$ Errikos Ntynan Hospital Center \\ ${ }^{2}$ Maastricht Universitair Medisch Centrum+ \\ ${ }^{3}$ Azienda Sanitaria Locale 3 Pescara
}

December 29, 2021

\begin{abstract}
Background and aim of the study. Wrapping of the ascending aorta (AA), isolated or associated with aortoplasty, has never been completely accepted. Some complications, as folding of the aortic wall, compression of the vasa vasorum and changes in the flow pattern, with consequent dilatation of the proximal arch, have been described. We used fresh autologous pericardium (FAP), so far never reported, to wrap the AA, with the aim to stabilize its size when moderately dilated, maintaining the preoperative dimension or limiting the reduction to a few mm. Material and Methods. From 2015 to 2019,10 patients, who were operated on for valve or coronary surgery or both, underwent wrapping of the AA with FAP. Mean age was $69 \pm 7$ years and ESII 3.5 \pm 1.7 . Four patients had moderately impaired ejection fraction (35-49\%). Results. There was no early or late mortality. One patient was reoperated on after 48 months for severe mitral regurgitation. At a follow up of $53 \pm 14$ months, a transthoracic echocardiogram showed that the AA size reduced slightly but significantly, from $45.2 \pm 2.0$ to $42.5 \pm 4.1 \mathrm{~mm}$, $\mathrm{p}=0.03$. The diameter of the proximal arch remained unchanged, from $37.1 \pm 1.6$ to $36.3 \pm 2.9 \mathrm{~mm}$, $\mathrm{p}=0.20$. Conclusions. In presence of moderately dilated AA wrapping can be a reasonable option. The use of FAP stabilizes the size of the aorta after a follow up of 53 months. Maintaining a size similar to the preoperative one avoids the complications related to the procedure.
\end{abstract}

\section{INTRODUCTION}

In the early period of cardiac surgery pump time and ischemic time were strictly correlated to perioperative mortality. The consequence was that all the efforts were targeted to reduce the duration of these procedural parts. The ascending aorta (AA) aneurysms were treated by wall plication, or aortoplasty, or wrapping or a mixture of them ${ }^{1-6}$. This experience was maintained over time, even if the analysis of the long-term results sometimes led to some concerns. Among these techniques, external wrapping was used first to support the ascending aorta after reduction aortoplasty, but later it became more popular as the only tool to reduce the AA size, as it does not need extracorporeal circulation. The concept of wrapping as a support without aortoplasty has been recently revisited by Golesworthy et $\mathrm{al}^{7}$, who introduced the personalized external aortic root support, developed as an alternative method to prevent dilatation of the aortic root in patients with Marfan's syndrome. However, this device is based on computer-aided design and 3D printing of the individual's AA on which the supporting mesh (microporous polyethylene terephthalate) is manufactured. It is a pre-emptive operations, addressed in general to patients with aortopathies with a AA size from 40 $\mathrm{mm}$ to not more than $60 \mathrm{~mm}^{8}$.

The material used for conventional wrapping of the ascending aorta (WAA) is a microporous polyethylene terephthalate (dacron) tubular graft, which is rigid and changes the ascending aorta in a tubular pipe. We decided to use the fresh autologous pericardium (FAP) not to reduce the AA diameter, but to stabilize its 
dimension to more or less the same at the baseline. The rationale was that FAP had some elasticity, that allowed the AA to adapt to changes in hemodynamic stresses, does not calcify or retract, as it is not inside the bloodstream, and it is able to adapt to the AA shape. We herein report the early and late results of our experience.

\section{MATERIAL AND METHODS}

From 2015 to 2019 FAP was used for wrapping of the AA in 10 patients. In all of them the AA dimensions were below $50 \mathrm{~mm}$. As none of them had bicuspid aortic valve or Marfan's syndrome, current guidelines did not advise AA replacement ${ }^{9}$. Preoperative clinical transthoracic echocardiographic data, with particular regards to aortic root at Valsalva sinuses level, sinotubular junction (STJ), AA and proximal arch dimensions, were collected in all patients using the parasternal long axis projection. Retrospective analysis of our database was approved by the Local Institutional Review Board, which waived patient consent.

Surgical technique. The pericardium was carefully dissected toward both pleuras. A piece large in general $16 \mathrm{~cm}$ and as long as the AA, from the origin of the brachiocephalic trunk to the STJ, was harvested. The pericardium was trimmed to fit the concavity and the convexity of the AA and was then kept in a bowl with saline. At the end of the procedure, after protamine, the AA was dissected for its whole length and surrounded by the pericardium. It was then sutured proximally and distally to the STJ and to the aorta close to the proximal arch with 4/0 Prolene, trying not to go through the full thickness of the aorta. The pericardium was then sutured around the aorta with $4 / 0$ Prolene and the excess of tissue cut (fig. 1A). Another suture (4/0 Prolene) is then passed parallel to the previous one, from the proximal to the distal AA (fig. 1B). This suture, after pressure reduction, either pharmacological or by means of intermittent closure of inferior vena cava, is put in tension to allow the pericardium to fit properly to the AA (fig. 1C). The final result is an AA with a size more or less similar to the preoperative one, but wrapped completely with pericardium from the STJ junction to the proximal arch (fig. 1D). In all patients who underwent myocardial revascularization, we used anaortic grafting, using as blood source the left, the right or both internal thoracic arteries.

Follow up . All patients were clinically followed at our outpatient clinic. The follow up ended on November 2021 and was 100\% complete. All the patients had an intermediate echocardiography after a mean of $16 \pm 4$ months. The final postoperative echocardiogram was performed between June and October 2021. Mean clinical and echocardiographic follow up was $53 \pm 14$ months.

Statistical analysis . Categorical variables are expressed as counts and percentages. Distribution of continuous variables was assessed using the Shapiro-Wilk test. Continuous variables are expressed as mean +standard deviation if normally distributed, as median with the 25 th and $75^{\text {th }}$ percentiles if not normally distributed. Pre- and postoperative data of each group were compared by paired t-test for normally distributed. variables, or Wilkinson test for non-normally distributed variables. The ANOVA test for repeated measures was applied for repeated echocardiographic data. If not otherwise indicated definition of the variables follows the definition reported in the EuroSCORE II model ${ }^{10}$. For all tests, a P-value $<0.05$ was considered statistically significant. The SPSS software (SPSS Inc, Chicago, IL) was used.

\section{RESULTS}

Table 1 shows some preoperative and perioperative data of the patients. Eight patients had chronic renal failure, mainly moderate $(n=7)$. Ejection fraction was good in most of the patients $(n=6)$. Nine patients had anaortic $\mathrm{CABG}$, isolated $(\mathrm{n}=4)$ or associated with aortic valve replacement $(\mathrm{AVR}, \mathrm{n}=5)$, whereas one patient had isolated AVR. Wrapping was successfully performed in all the patients.

There was no early or late mortality and no complications, either during the postoperative period or during the follow up. One patient was reoperated on after 48 months because of severe mitral valve disease and underwent uneventful mitral replacement.

Echocardiographic outcome . Table 2 shows the results of the median and last echocardiography compared with baseline. The ANOVA for repeated measurements showed that the aortic root at the level of 
the Valsalva sinuses reduced slightly but significantly as well as the AA diameter $(\mathrm{p}=0.03)$. The STJ and proximal arch showed a mild reduction, but not significant. Comparison between the median and the last follow up showed that all the results remained stable over time.

\section{DISCUSSION}

Wrapping of the AA was first described by Bahnson and Nelson in $1956^{1}$, who performed aortic AA reduction using a side clamp and wrapping in 2 patients. Both suddenly died 4 and 5 months after surgery. Wrapping in the early period was associated with oval excision of the AA (aortoplasty) and was only an external support and not a tool to reduce the size of the aorta ${ }^{11}$. Results of aortoplasty and wrapping were satisfying ${ }^{12,13}$, but a meta-analysis of Plonek et $\mathrm{al}^{14}$ with 722 cases concluded that isolated aortic wrapping had better results than wrapping and aortoplasty or wrapping and aortic plication. However, even if wrapping of the AA is technically simple, the report of complications intrinsic to the technique with/out aortoplasty prevented a wide application of this strategy ${ }^{15-21}$. In contrast, replacement of the AA is a well-studied procedure, reproduceable and easy to perform, with uniformly good results. Wrapping of the AA involves the use of a conventional vascular prosthesis of a preselected size, smaller than that of the AA, with the aim to avoid further dilatation of the aorta and the related complications. A problem is the formation of folds ${ }^{22}$. The size of a vascular graft is, at maximum, $36 \mathrm{~mm}$. Then the reduction is at least $10 \mathrm{~mm}$ or more. The presence of folds, due to a severe mismatch between the graft and the size of the AA, changes the flow patterns and can lead to compression and erosion of the aortic wall. Neri et $\mathrm{al}^{15}$, in 2 patients who underwent a reoperation, found that the reinforced aorta was significantly thinner, with a sclerotic microstructure in which layers were no longer present. Also, the atrophied aortic wall showed cellular and neovascular infiltration and common in a foreign-body reaction. On the contrary, the samples retrieved from the non-reinforced aorta showed a basically normal histologic structure. At the basis of these changes there are the compromise of the vasa vasorum that nourish the middle layer of the aorta, chronic inflammatory response to the foreign material, and sustained compression of the aortic wall between the pressure of blood and of the external prosthesis. As a consequence of these changes, the aorta becomes a passive conduit with biomechanical characteristics similar to those of a synthetic vascular prosthesis. These changes seem to be less evident when the AA is reinforced with a dacron (macro-porous) mesh instead of a vascular conduit ${ }^{23}$, probably due to its greater elasticity.

An external support that reinforces the vessel without interfering with the pattern of flow is very attractive, but not free of drawbacks. The aorta is a very strong structure. Aortic rupture does not occur if the intraluminal pressure ranges from 790 to $2070 \mathrm{~mm} \mathrm{Hg}{ }^{24}$. Robertson and Smith, injecting water into the media of 42 fresh human aortas, found that the lowest pressure required to exceed the cohesive strength of the media was $273 \mathrm{~mm} \mathrm{Hg}$ and the highest was $975 \mathrm{~mm} \mathrm{Hg}$, with a mean of $566 \mathrm{~mm} \mathrm{Hg}^{25}$. Therefore, the aorta is highly resistant to rupture or dissection. Recently, an appealing theory on the genesis of intramural hematoma and aortic dissection was reproposed ${ }^{26,27}$. Vasa vasorum dysfunction is the link between these entities, which are seen as progression of one into the other. Rupture and bleeding of the vasa vasorum into the media are the causes of intramural hematoma. It can remain limited to the thickness of the aorta or cause an intimal tear, which is at the basis of classic aortic dissection. The vasa vasorum fill during diastole as in the coronary circulation. Thus, an increase in arterial diastolic pressure results in reduced perfusion ${ }^{28}$, which can cause vessel wall hypoxia and neoangiogenesis, with the neovessels more fragile and prone to bleed. Hypertension also can reduce blood flow by distortion or compression of the vasa, generating changes in the walls of the vasa vasorum with critical ischemia and necrosis of the media. Other factors (eg, inflammation) can induce aberrant and adverse remodelling of the aortic wall, including smooth muscle cell loss in the media and extracellular matrix degradation in the media and the adventitia. The consequence is chronic dilation of the aorta, but an acute aortic syndrome can superimpose at any moment. Increased tension or chronic inflammation due to wrapping can cause localized hematoma or necrosis, weakening the aortic wall. The benefit of reducing wall stress reducing the diameter has to be compared with the interference with the nourishment of the aortic wall.

Wrapping also modifies the biomechanical characteristics of the blood flow in the entire arterial system ${ }^{29}$. 
The synthetic materials used to externally reinforce the aorta are much less elastic than a healthy or diseased native aorta, which alters the pressure of the entire arterial system. This fact has been associated with a higher risk of cardiovascular events in hypertensive patients ${ }^{30}$. On the other hand, the compliance mismatch between the native and the reinforced aorta leads to hemodynamic changes that increase the circumferential wall stress in the aortic segments at the interface of supported and unsupported aorta ${ }^{29}$, shifting distally the area of greatest expansion ${ }^{31}$. This particular aspect was emphasized in a recent paper from Kim et al ${ }^{32}$, where the Authors compared the long term outcome of wrapping compared to conventional replacement of the AA in propensity matched patients. After a median follow up of 7.1 years, the proximal arch dilated in the wrapping group $(0.343 \mathrm{~mm} /$ year $)$, but not in the replacement group. In the wrapping group preoperative AA size was a risk factor for proximal arch dilatation, with a cut off value of $47.15 \mathrm{~mm}$. Total adverse aortic event (reoperation of ascending or arch aorta, dissection or rupture, redilatation of the aortic arch) were higher in the wrapping group $(24.3 \%$ vs $14.3 \%, \mathrm{p}=0.010)$ during the follow up. Then, graft migration because of proximally or distally incomplete fixation of the prosthesis can cause proximal and distal redilatation, but proximal arch dilatation can be due to mechanisms intrinsic to the concept of wrapping even if presence of a correct surgical technique.

We decided, in presence of a moderately dilated AA, to change the strategy of wrapping. The size of the AA remained unchanged or reduced only of a few $\mathrm{mm}$ (fig. 1E), to avoid all the previously described possible complications, in particular folds and vasa vasorum compression. The use of FAP allowed us to follow the natural curvature of the vessel and to adapt proportionally to all the different portions of the aorta (fig. 1D).

Moreover, it was observed that FAP is able to stretch in its two axes ${ }^{33}$ and that the systolic deformation of the epicardial surface is characterized by a longitudinal shortening of $10 \%^{34}$. This property can act as shock absorber during the changes of the intra-aortic pressure, avoiding the aorta to become a static and passive conduit.

As our target is to stabilize the ascending aorta, the aorta does not fold internally and the hemodynamic stress at the distal portion of the AA remains unchanged. The concept we applied is similar to that one reported by Robicseck et $\mathrm{al}^{35}$ who described the use of a tubular dacron prosthesis, if necessary enlarged by a dacron oval patch, to reinforce the aortic wall, procedure that the Authors called "external grafting". The targets were patients with a moderately dilated AA, "too big to leave in and too small to take out", as the population by us treated.

Our results have been good in all patients, without any early or late aortic complication. The AA size remained stable with time. However, the main limitation of this report is the small number of patients, only 10, even if with a reasonable follow up. Moreover, the follow up was performed with echocardiographic parameters, as many of the patients had no preoperative CT scan and an echocardiogram was easy to perform.

In conclusion, we think that wrapping of the AA (from the STJ till the beginning of the proximal arch) can have still a place when the AA is moderately dilated. The use of FAP allows to avoid further dilatation, maintaining more or less the same diameter at the moment of wrapping, without interfering with the nourishment of the aortic wall and with the hemodynamic patterns. The rationale of wrapping has to be changed, from reduction to values more or less normal (by 10 or more $\mathrm{mm}$ ) to pure stabilization of the actual diameter to avoid any further dilatation.

\section{LEGEND}

Figure $1-A$, after being sutured distally (at the level of the sino-tubular junction) and proximally (between the distal ascending aorta and the proximal arch), the fresh autologous pericardium is wrapped around the aorta. B, another suture is then passed parallel to the previous one (white arrows and dashed lines), from the proximal to the distal ascending aorta (fig. 1B). C, this suture is put in tension to allow the pericardium to fit properly to the ascending aorta. D, the final aspect is an ascending aorta with the same shape and size than before surgery, but completely wrapped with fresh autologous pericardium, that, at the follow up, becomes completely adherent to the aorta $(\mathrm{E})$. 
Table 1 - Preoperative and perioperative data $(n=10)$

Age (y) 69+-7

Female gender 2

Hypertension 7

Diabetes 0

COPD 0

Creatinine $1+-0.2$

Creatinine clearance $(\mathrm{ml} / \mathrm{min}) 78+-28$

CRF moderate 7

Severe 1

Peripheral vascular disease 1

Previous CVA 0

Redo 0

NYHA Class I-II 7

III 3

ESII $3.5+-1.7$

$\mathrm{EF}(\%)[?] 506$

35-49 4

AVR 1

AVR+CABG 5

CABG (off-pump) 4

ECC (min, n=6) 132+-26

Xclamping (min, $\mathrm{n}=6$ ) 88+-19

Table 2 - Echocardiographic aortic measurements before surgery, at intermediate and at last follow up.

baseline intermediate last p1 p2 p3 ANOVA

$16+-4$ mos $53+-14$ mos

Valsalva sinuses (mm) 37.4+-1.9 36.5+-2.6 36.2+-2.7 0.040 .030 .080 .03

Sinotubular junction (mm) 38.5+-2.3 37.7+-3.0 37.7+-2.9 $0.07 \quad 0.070 .340 .07$

Ascending aorta (mm) 45.2+-2.0 42.7+-4.2 42.5+-4.1 0.040 .030 .330 .03

Proximal arch (mm) 37.1+-1.6 36.6+-2.5 36.3+-2.9 0.320 .200 .080 .20

p1: baseline vs median 1: p2: baseline vs last; p3: median vs last

Legend: mos, months.

\section{REFERENCES}

1. Bahnson HT, Nelson AR. Cystic medial necrosis as a cause of localized aortic aneurysms amenable to surgical treatment. Ann Surg 1956;144:519-29. 
2. Robicsek F, Daugherty HK, Mullen DC, et al. Long-range observations with external aortic grafts. J Cardiovasc Surg (Torino) 1976;17:195-201.

3. Robicsek F. A new method to treat fusiform aneurysms of the ascending aorta associated with aortic valve disease: an alternative to radical resection. Ann Thorac Surg 1982;34:92-4.

4. Baumgartner F, Omari B, Pak S, Ginzton L, Shapiro S, Milliken J. Reduction aortoplasty for moderately sized ascending aortic aneurysms. J Card Surg 1998;13:129-32.

5. Walker T, Bail DH, Gruler M, Vonthein R, Steger V, Ziemer G. Unsupported reduction ascending aortoplasty: fate of diameter and of Windkessel function. Ann Thorac Surg 2007;83:1047-53.

6. Arsan S, Akgun S, Kurtoglu N, Yildirim T, Tekinsoy B. Reduction aortoplasty and external wrapping for moderately sized tubular ascending aortic aneurysm with concomitant operations. Ann Thorac Surg 2004;78:858-61.

7. Golesworthy T, Lamperth M, Mohiaddin R, Pepper J, Thornton W, Treasure T. The Tailor of Gloucester: a jacket for the Marfan's aorta. Lancet 2004;364:1582.

8. Nemec P, Pepper J, Fila P. Personalized external aortic root support. Interact Cardiovasc Thorac Surg 2020;31:342-5.

9. Erbel R, Aboyans V, Boileau C, et al. 2014 ESC Guidelines on the diagnosis and treatment of aortic diseases: Document covering acute and chronic aortic diseases of the thoracic and abdominal aorta of the adult. The Task Force for the Diagnosis and Treatment of Aortic Diseases of the European Society of Cardiology (ESC). Eur Heart J 2014;35:2873-926.

10. Nashef SA, Roques F, Sharples LD, et al. EuroSCORE II. Eur J Cardiothorac Surg 2012;41:734-44; discussion 44-5.

11. Robicsek F, Thubrikar MJ. Conservative operation in the management of annular dilatation and ascending aortic aneurysm. Ann Thorac Surg 1994;57:1672-4.

12. Carrel T, von Segesser L, Jenni R, et al. Dealing with dilated ascending aorta during aortic valve replacement: advantages of conservative surgical approach. Eur J Cardiothorac Surg 1991;5:137-43.

13. Belov IV, Stepanenko AB, Gens AP, Savichev DD, Charchyan ER. Reduction aortoplasty for ascending aortic aneurysm: a 14-year experience. Asian Cardiovasc Thorac Ann 2009;17:162-6.

14. Plonek T. A meta analysis and systematic review of wrapping of the ascending aorta. J Card Surg 2014;29:809-15.

15. Neri E, Massetti M, Tanganelli P, et al. Is it only a mechanical matter? Histologic modifications of the aorta underlying external banding. J Thorac Cardiovasc Surg 1999;118:1116-8.

16. Akgun S, Atalan N, Fazliogullari O, Kunt AT, Basaran C, Arsan S. Aortic root aneurysm after off-pump reduction aortoplasty. Ann Thorac Surg 2010;90:e69-70.

17. Bauer M, Grauhan O, Hetzer R. Dislocated wrap after previous reduction aortoplasty causes erosion of the ascending aorta. Ann Thorac Surg 2003;75:583-4.

18. Dhillon JS, Randhawa GK, Straehley CJ, McNamara JJ. Late rupture after dacron wrapping of aortic aneurysms. Circulation 1986;74:I11-4.

19. Doyle M, Peeceeyan S, Bonar F, Horton M. Rarefaction of the aorta under Dacron wrap: a rare complication. Interact Cardiovasc Thorac Surg 2014;19:341-3.

20. Gonzalez-Santos JM, Arnaiz-Garcia ME. Wrapping of the ascending aorta revisited-is there any role left for conservative treatment of ascending aortic aneurysm? J Thorac Dis 2017;9:S488-S97. 
21. Ehrlich T, Federspiel JM, Bohle RM, Schafers HJ. Severe aortic wall degeneration after aortic wrapping. Eur J Cardiothorac Surg 2021;60:425-7.

22. Plonek T, Dumanski A, Nowicki R, Kustrzycki W. Computed tomography angiography of aorta subjected to external wrapping. J Cardiothorac Surg 2016;11:89.

23. Cohen O, Odim J, De la Zerda D, et al. Long-term experience of girdling the ascending aorta with Dacron mesh as definitive treatment for aneurysmal dilation. Ann Thorac Surg 2007;83:S780-4; discussion S5-90.

24. Oppenheim F. Gibt es eine spontanruptur der gesunden aorta und wie kommt sie zustande? Munchen Med Wchnschr 65;1234-7, 1928.

25. Robertson JS, Smith KV. An analysis of certain factors associated with the production of experimental dissection of the aortic media, in relation to the pathogenesis of dissecting aneurysm. J Pathol Bacteriol 1948;60:43-9.

26. Haverich A, Boyle EC. Aortic dissection is a disease of the vasa vasorum. JTCVS open 2021;5:30-2.

27. Calafiore AM, Katsavrias K, Di Marco M, Guarracini S, Di Mauro M. Commentary: Vasa vasorum dysfunction and acute aortic syndromes: when guidelines do not follow the evolution of knowledge. JTCVS open 2021:5:33-4.

28. Scotland RS, Vallance PJ, Ahluwalia A. Endogenous factors involved in regulation of tone of arterial vasa vasorum: implications for conduit vessel physiology. Cardiovascular research 2000;46:403-11.

29. Izgi C, Newsome S, Alpendurada F, et al. External Aortic Root Support to Prevent Aortic Dilatation in Patients With Marfan Syndrome. J Am Coll Cardiol 2018;72:1095-105.

30. Schiffrin EL. Vascular stiffening and arterial compliance. Implications for systolic blood pressure. Am J Hypertens 2004;17:39S-48S.

31. Singh SD, Xu XY, Pepper JR, Izgi C, Treasure T, Mohiaddin RH. Effects of aortic root motion on wall stress in the Marfan aorta before and after personalised aortic root support (PEARS) surgery. J Biomech 2016;49:2076-84.

32. Kim HH, Lee S, Lee SH, Youn YN, Yoo KJ, Joo HC. The long-term fate of ascending aorta aneurysm after wrapping versus replacement. J Thorac Cardiovasc Surg 2021.

33. Pavan PG, Pachera P, Tiengo C, Natali AN. Biomechanical behavior of pericardial human tissue: a constitutive formulation. Proc Inst Mech Eng H 2014;228:926-34.

34. Wen H, Bennett E, Epstein N, Plehn J. Magnetic resonance imaging assessment of myocardial elastic modulus and viscosity using displacement imaging and phase-contrast velocity mapping. Magn Reson Med 2005;54:538-48.

35. Robicsek F, Daugherty K, Mullen DC, Harbold NB, Jr., Masters TN. Is there a place for wall reinforcement in modern aortic surgery? Arch Surg 1972;105:827-9. 


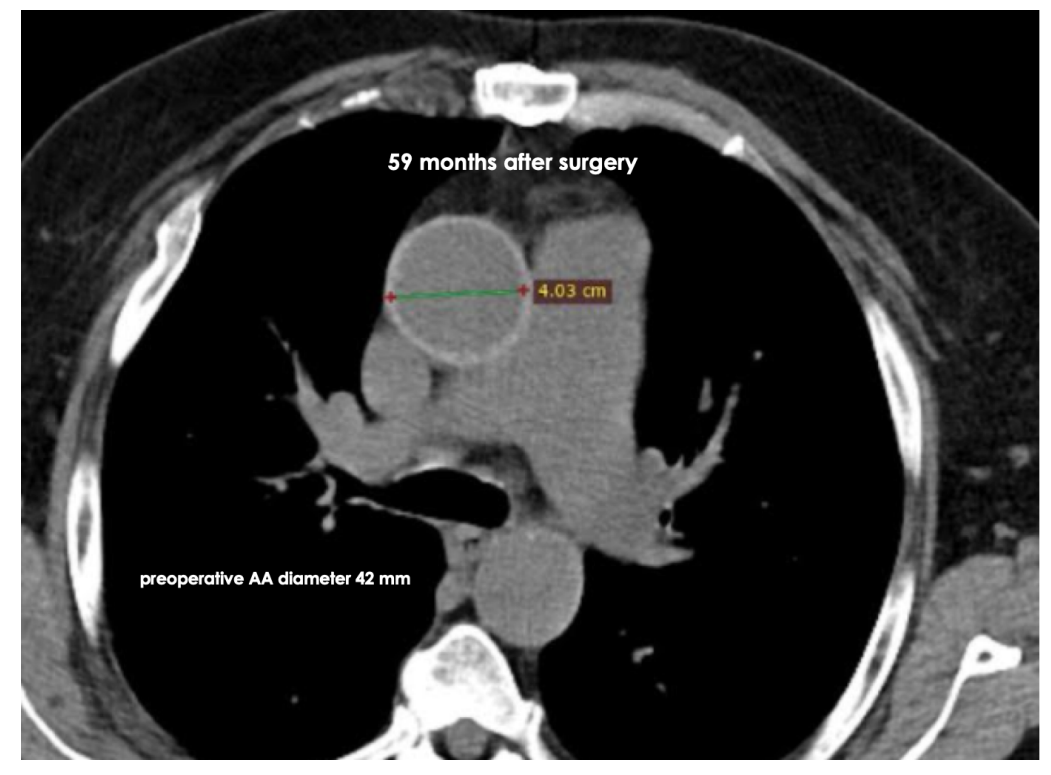

Fig. $1 E$

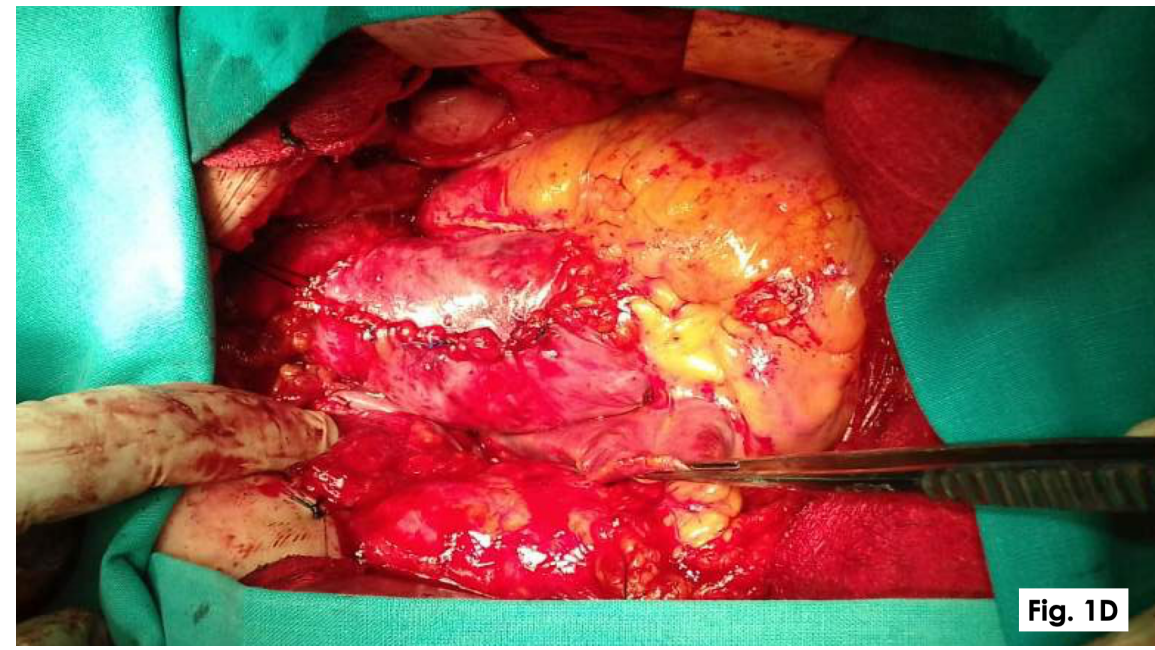



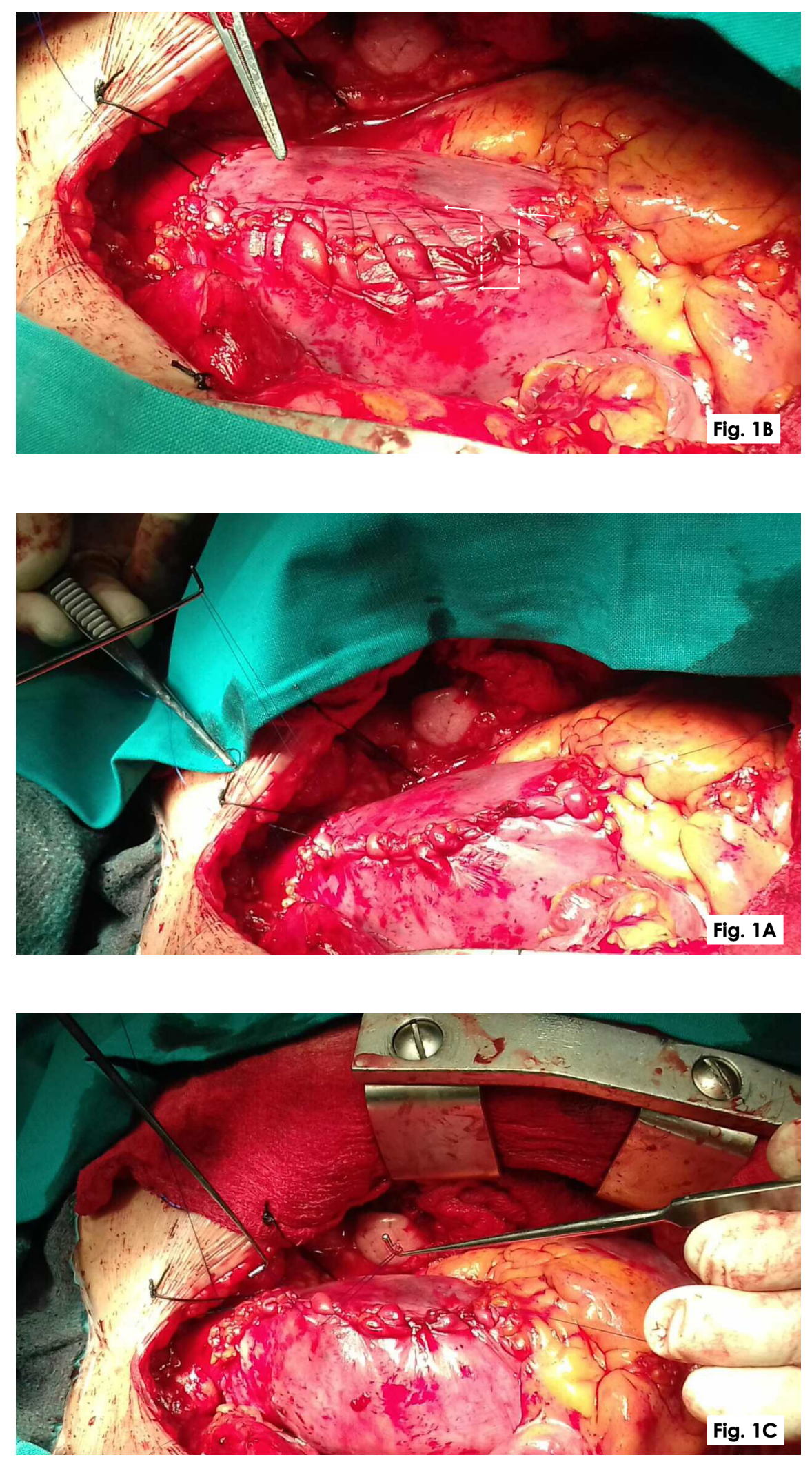